\title{
Computed Tomography Osteoabsorptiometry Is Reliable for the Determination of the Subchondral Bone Mineralization Distribution in the Rabbit Knee
}

\author{
H. Anetzberger ${ }^{\mathrm{a}} \quad$ A. Mayer ${ }^{\mathrm{b}} \quad$ C.U. Schulz ${ }^{\mathrm{a}} \quad$ M. Müller-Gerbl ${ }^{\mathrm{c}}$ \\ ${ }^{\text {a } O r t h o p a ̈ d i s c h e ~ G e m e i n s c h a f t s p r a x i s ~ u n d ~}{ }^{\mathrm{b}}$ Abteilung Physikalische Medizin und Frührehabilitation, \\ Klinikum Bogenhausen, München, Germany; ${ }^{~}$ Institute of Anatomy, Basel, Switzerland
}

\section{Key Words}

Subchondral bone mineralization - Rabbit knee •

Osteoarthritis

\begin{abstract}
Rabbits are among the most frequently used animals in osteoarthritis research. It is meanwhile accepted that the subchondral bone plate (SBP) plays a key role in the development of osteoarthritis. The most suitable technique for analyzing subchondral bone mineralization is computed tomography osteoabsorptiometry (CT-OAM). Because CTOAM has not yet been applied to smaller animals, the purpose of this study is to test the reliability of CT-OAM in the rabbit knee. Another important task in animal experiments is the intra- and interindividual difference of the measurement parameters. Our hypothesis is that there is no difference regarding both the position of the density maxima and the bone mineral density (BMD) of the SBP comparing right and left tibial plateaus of rabbits. For evaluating the reliability, a rabbit knee was examined by computed tomography 6 times at weekly intervals. The subchondral mineralization distribution was measured by means of CT-OAM. Positions of the density maxima and BMD of the SBP were determined in a standardized procedure. Furthermore, both parameters were evaluated in 6 female White New Zealand rabbits. Positions of density maxima and BMD in the SBP in left tibial pla-
\end{abstract}

teaus were compared with right tibial plateaus. The relative coefficient of variation as a parameter for reproducibility was $1.6 \%$ for determining the position of the density maxima and $1.2 \%$ for measuring the BMD. The positions of density maxima and relative BMD between right and left tibial plateaus varied only about $2 \%$ intraindividually, whereas interindividual variance was about $10 \%$. In conclusion, determination of the position of density maxima as well as BMD of the SBP by means of CT-OAM is reliable and reproducible in the rabbit knee. We recommend using the contralateral limb as control, because intraindividual accordance of the mineralization patterns and of the BMD of the SBP was higher than interindividual accordance.

Copyright $\odot 2012$ S. Karger AG, Basel

\section{Introduction}

Previous research has clearly pointed out that the subchondral bone adapts according to the mechanical loading [1-3]. Looking at more detail, a distinction has to be made between various morphological parameters. Subchondral bone mineral density, mineralization pattern of the subchondral bone plate (SBP), thickness of the SBP, trabecular thickness and trabecular connectivity have in common that they are shaped by mechanical load. From all these parameters, only the mineralization distribution

\section{KARGER}

Fax +41613061234 E-Mail karger@karger.ch www.karger.com

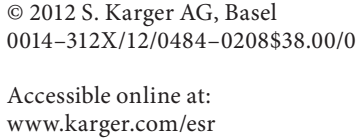

Hermann Anetzberger

Orthopädische Gemeinschaftspraxis

Hanauerstrasse 65

DE-80993 Munich (Germany)

E-Mail dr.anetzberger@orthospezial.de 
of the SBP is like a fingerprint and shows indirectly, as a morphological parameter, the long-term stress distribution of an individual joint [4-8].

The interrelation between the 'loading history' of a joint and the subchondral bone mineralization pattern is a key topic in mechanobiology research. For instance, the results of animal studies suggest that the morphological changes in the SBP play an important role in the induction of osteoarthrosis $[9,10]$. Currently, the most suitable method for quantifying the subchondral bone mineralization is computed tomography osteoabsorptiometry (CT-OAM) [2]. This method is based on the use of computed tomography (CT). The CT data were transferred into a computer workstation and processed using a dedicated software program (Analyze ${ }^{\circledR}$, Mayo Foundation, Rochester, Minn., USA). After manual selection of the SBP in each slice, the mineralization distribution of subchondral bone was displayed using a maximum intensity projection algorithm. For better visualization the bone pixels were assigned to 10 density value groups [range: 200-1200 Hounsfield units (HU)] represented by false colors. Thus, a surface representation of the 3-dimensional mineral density distribution of the SBP is obtained. Furthermore, by using a phantom reference plate the relative bone mineral density (BMD) of the SBP can be calculated. In contrast to dual-energy X-ray absorptiometry $[11,12]$ or micro-CT $[13,14]$, this method allows for a statistical analysis of both, the position of the density maxima and the relative BMD of the SBP.

Up to now the validity of this method had only been established for human joints [15-17] and large animals [9]. However, it has not yet been established whether the resolution of standard CT is sufficient to yield relevant data in small joints, as e.g. the rabbit knee. This is of particular interest, because the rabbit is one of the most common laboratory animals in osteoarthrosis research [18].

Therefore, the aim of the present study is to evaluate the reliability of CT-OAM for the rabbit knee. Because no data on the mineralization distribution in healthy rabbit knee joints existed, another goal was to evaluate the distribution of the subchond ral bone mineralization in tibial plateaus of rabbits. In experimental studies, the contralateral limb is often used as control. This is of great advantage, because intraindividual difference compared to interindividual difference allows the use of a smaller number of animals with the same statistical power [19]. Therefore, the hypothesis to be tested was that there is no difference in the position of density maxima and BMD of the SBP when comparing right and left articular surfaces.

\section{Materials und Methods}

For the present study, 12 knees from 6 adult female New Zealand White rabbits with a mean body weight of $3.60 \pm 0.44 \mathrm{~kg}$ (Charles River, Kisslegg, Germany) were collected. The tibial articular surfaces were evaluated after the articular capsule had been opened, to exclude cartilage lesions. The experimental work was performed after approval by the animal care committee of the Bavarian government, Munich, Germany.

\section{CT Osteoabsorptiometry}

The tibias were positioned in the CT scanner in such a way that the tibial plateau was parallel to the gantry aperture and the tibial tuberosity was located ventrally. One-millimeter sagittal slices were made from each knee joint $(\mathrm{n}=12)$, each of which was digitally imaged $(80 \mathrm{kV} ; 16 \mathrm{~mA}$; resolution: $0.125 \times 0.125 \mathrm{~mm})$ in a computer tomograph (Somatom Plus 4, Siemens, Erlangen, Germany). For all CT investigations, a reference phantom (Siemens, Erlangen, Germany) was used comprising two stable plastics, a water-equivalent standard and a bone-equivalent standard containing $200 \mathrm{mg}$ hydroxyapatite/ml). For ascertaining the reliability of CT-OAM, one knee joint was randomly selected and sectioned by means of CT 6 times at weekly intervals.

The CT data sets were then entered into a computer workstation and processed by means of an imaging program (Analyze, Mayo Foundation). First, the tibial plateau was selected in individual sections (fig. 1a) and subsequently reconstructed 3-dimensionally with the tibial articular surface viewed from above perpendicular to the frontal plane and perpendicular to the longitudinal axis of the tibia (fig. 1b). The rotation data were recorded. Then, the SBP was selected in all individual sections, and a further 3-dimensional reconstruction was made from the previously established rotation data (fig. 1c). The density distribution was processed by maximum-intensity projection so that for every image point each maximum density value of the underlying bone plate is projected onto the surface. This yields reproducible density patterns. The content of mineral salts is presented indirectly through variation in HU. For visualization, HU were summarized in steps of 100 and attributed to different colors (fig. 1d). Finally, the reconstructed 3-dimensional image of the articular surface and the colored density distribution were blended for better orientation.

For quantitative analysis of density maxima, all left articular surfaces were mirrored in order to allow for comparison. In order to allow comparison of joint surfaces with different sizes, a coordinate system capable of linear extension was selected and subdivided into 20 units. The tangent to the posterior cortex of the tibial plateau was defined as the $\mathrm{x}$-axis and the tangent to the medial tibial plateau as the y-axis. Finally, the coordinates of the areal centroid within the zone of the two highest density levels of each maximum were determined and evaluated statistically (fig. 1e).

For quantifying the BMD of the SBP, the medial and lateral articular surfaces of the tibia and femur were evaluated separately. By means of analytical photogrammetry, the number of pixels for each $\mathrm{HU}$ value was determined. Using the reference phantom, a calcium value could be algorithmically computed for each $\mathrm{HU}$ value. Thus, the relative calcium content of the SBP in relation to its volume (milligrams Ca per milliliter) was determined. 

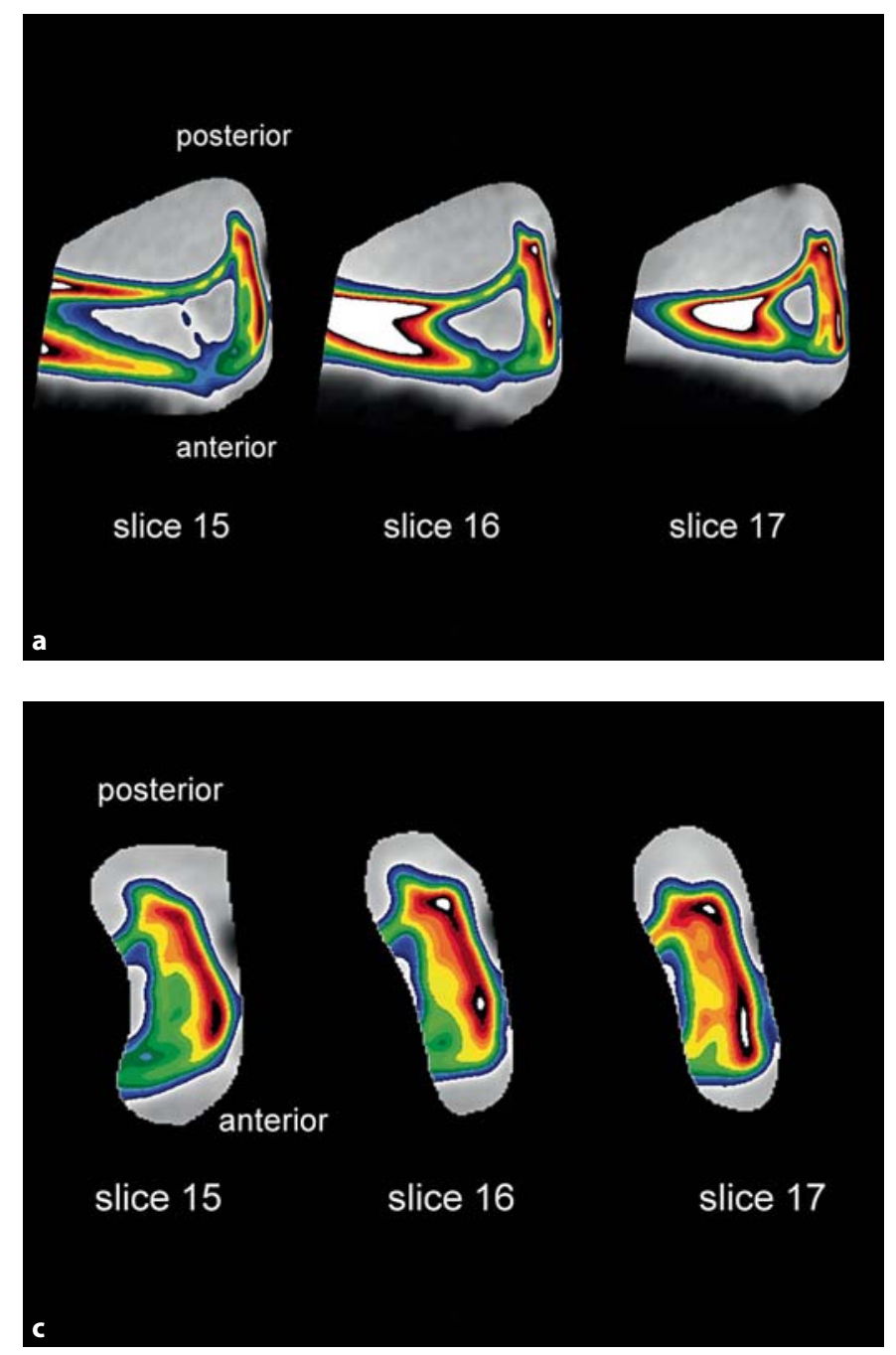

Fig. 1. Methodological procedure of CT-OAM. a Selection of the tibia in individual slices. $\mathbf{b}$ Three-dimensional reconstruction of the tibial plateau with the view from above. c Selective representation of the SBP in individual slices. $\mathbf{d}$ Three-dimensional reconstruction of the SBP and presentation of the mineralization distribution by colors. e Determining the coordinates of density maxima.
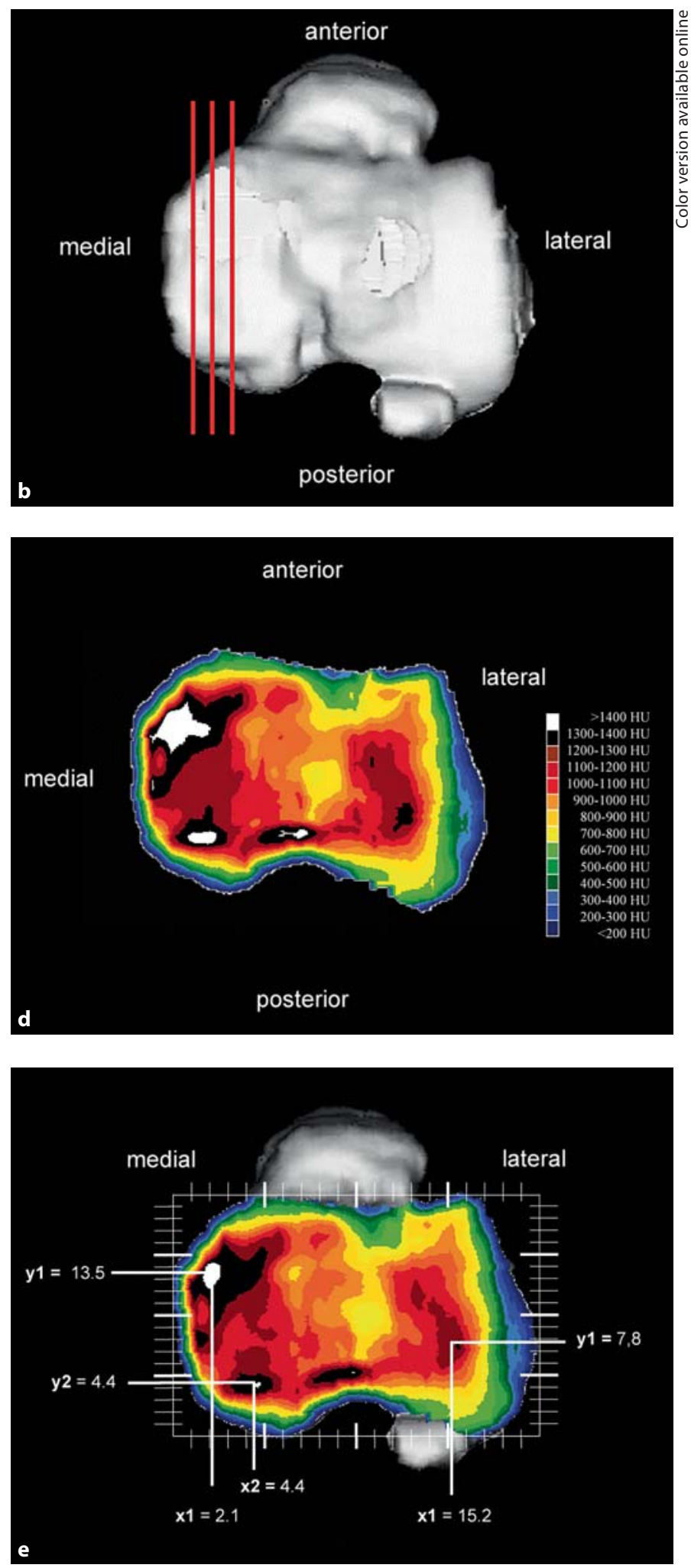


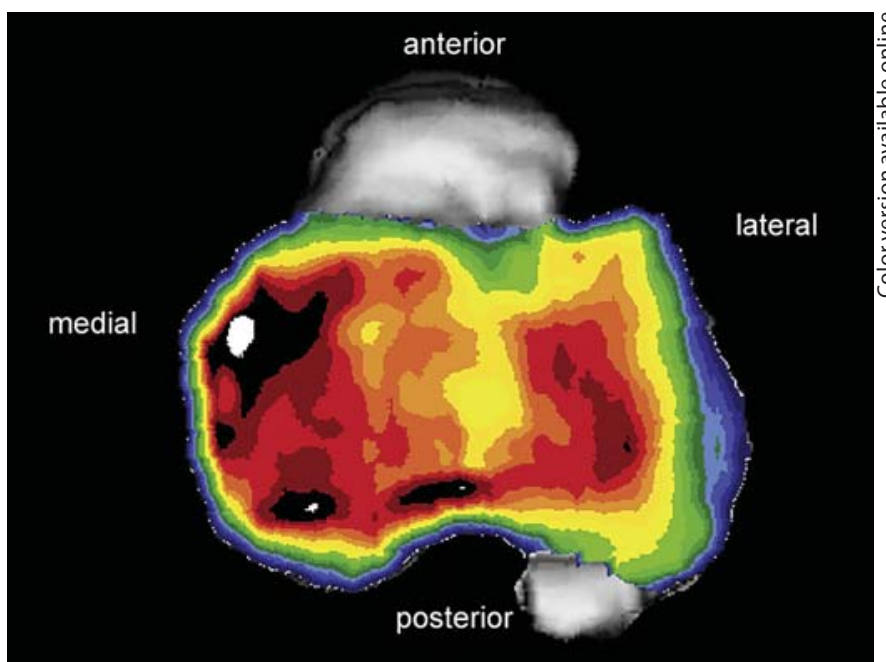

Fig. 2. Mineralization distribution of tibial SBP viewed from above. The medial surface has 2 density maxima; one of these is located anteriorly, the other is located posteriorly. The lateral articular surface contains only 1 maximum density area. The degree of mineralization declines toward the edge of the articular surface.

\section{Statistics}

The statistical analyses were carried out using the software SPSS for Windows, version 13.0 (SPSS Inc., Chicago, Ill., USA). All data are presented as mean value \pm standard deviation. The relative coefficient of variation in percent $\left(\right.$ rel. $\mathrm{CV}=\mathrm{CV} / \mathrm{n}^{0.5} \times 100$ ) was used to characterize the precision of repeated measurements. For analysis of side-to-side differences, the Wilcoxon signed-rank test was applied. The level of significance selected was $p<0.05$

\section{Results}

\section{Reproducibility of the Method and Position of Density}

\section{Maxima}

On the tibial plateau of the rabbit we always observed 3 density maxima. Within the articular surface, they are located centrally in the area not covered by a meniscus. On the lateral tibial joint surface there was only 1 density maximum which was located centrally. In contrast to this we found on the medial tibial joint surface 2 density maxima, one of them was located anteriorly and the other posteriorly. The degree of mineralization declines toward the edge of the articular surface (fig. 2).

The relative coefficient of variation after 6 repeated measurements for the lateral density maximum was $0.3 \%$ (x1lat) and $0.9 \%$ (yllat). The relative coefficient of variation for the medial anterior density maximum was $1.4 \%$ (xlmed) and $0.5 \%$ (y1med), for the medial posterior density maximum $0.3 \%$ (x2med) and $0.9 \%$ (ylmed), respectively.

Subchondral Bone Mineralization in the Rabbit Knee

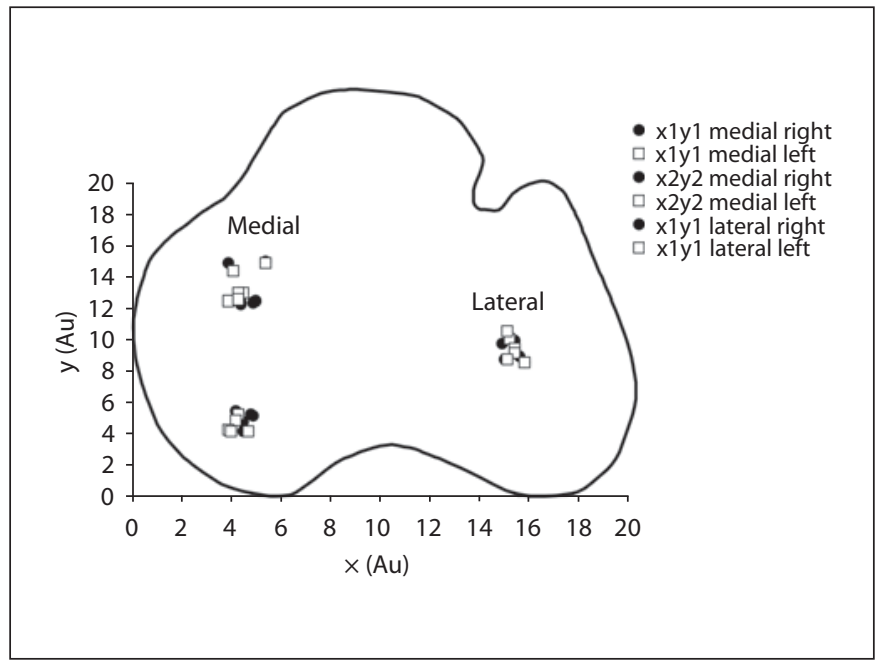

Fig. 3. Location density maximum in the coordinate system. A comparison of the position of density maxima $(n=6)$ in the medial and lateral articular surface showed no difference between the right and the left sides $(\mathrm{p}<0.05)$.

The position of density maxima in the medial and lateral tibial articular surfaces defined by coordinates was identical when comparing left and right sides (fig. 3). The coefficient of variation for the position of maximum density maxima for an intraindividual comparison was $1.1 \%$ for the lateral density maximum, 2.3\% for the medial anterior maximum, and $4.5 \%$ for the medial posterior density maximum. The coefficient of variation for interindividual comparison calculated as $4.6 \%$ for the lateral density maximum, $9.8 \%$ for the medial anterior density maximum and $9.3 \%$ for the medial posterior maximum, respectively.

\section{Relative BMD of the SBP}

The relative coefficient of variation for the repeated determination of BMD of the SBP was $1.2 \%$ for the medial tibial plateau and $0.9 \%$ for the lateral tibial plateau.

The mean relative BMD of the medial SBP was $57 \pm$ $5 \mathrm{mg} \mathrm{Ca} / \mathrm{ml}$ on the right side and $56 \pm 6 \mathrm{mg} \mathrm{Ca} / \mathrm{ml}$ on the left side. The mean relative BMD of the lateral SBP was $52 \pm 5 \mathrm{mg} \mathrm{Ca} / \mathrm{ml}$ on the right side and $51 \pm 5 \mathrm{mg}$ $\mathrm{Ca} / \mathrm{ml}$ on the left side. While there is no statistically significant difference in BMD between the right and left, BMD of the SBP is significantly higher in the medial tibial plateau compared to the lateral tibial plateau (fig. 4).

The coefficient of variation for intraindividual comparison was $2.0 \%$ for the medial tibial plateau and $2.4 \%$ 


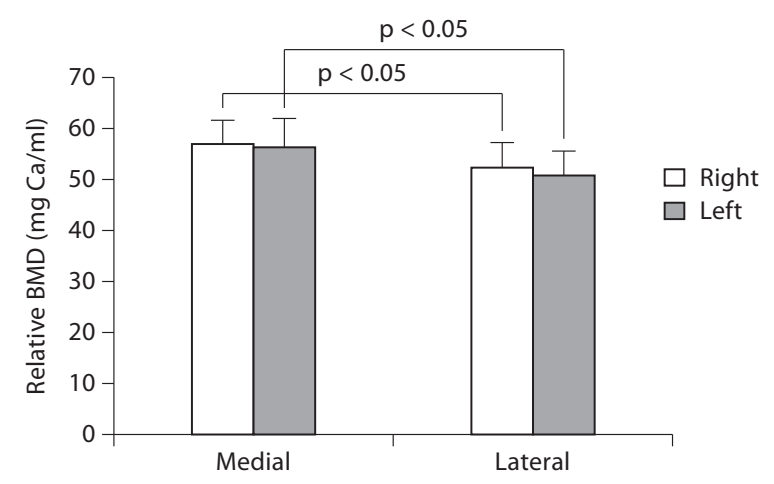

Fig. 4. Relative BMD of the medial and lateral tibial SBP. The BMD is significantly higher in the medial tibial plateau compared to the lateral tibial plateau. The BMD in right and left medial and lateral SBP is identical.

for the lateral tibial plateau, respectively. The coefficient of variation for interindividual comparison was $9.0 \%$ for the medial tibial plateau and $9.6 \%$ for the lateral tibial plateau, respectively.

\section{Discussion}

Our study clearly shows that CT-OAM yields reproducible and reliable data of subchondral mineralization distribution also in smaller joints, such as the rabbit knee. Both the position of the density maxima and the relative $\mathrm{BMD}$ of the SBP can be reliably determined. Furthermore we were able to demonstrate that the position of density maxima and relative BMD between right and left tibial plateaus of one individual varied only by about $2 \%$, whereas interindividual variance was about $10 \%$. In consequence we recommend the use of the contralateral limb as control in an experimental setup.

Previous research has shown that the mineralization distribution of the SBP is like a fingerprint and shows indirectly as a morphological parameter the long-term stress distribution of an individual joint [4-8]. This means that the areas of highest load are also the areas of highest bone mineralization. CT-OAM is the most elegant method to visualize and to quantify subchondral bone mineralization distribution [5]. We were able to demonstrate that this method using a conventional computer tomograph (Somatom Plus 4, Siemens) with a resolution of $0.125 \times 0.125 \mathrm{~mm}$ and a slice thickness of $1 \mathrm{~mm}$ generates reproducible densitograms of subchondral bone mineralization also in small joints. The relative coefficient of variation for 6 repeated measurements and 6 repeated determinations of the position of the density maxima was less than $1.5 \%$. The same was true for measuring the relative BMD of the SBP, revealing a coefficient of variation even below $1.2 \%$. Therefore, methodological errors due to variation of the section plane, manual selection of bone density or determining the position of maximum density through the area centers are extremely small. There are some further possible sources of error that require discussion. First, we performed the CT-OAM at 'maximum intensity projection', which projects the highest density voxels from the subchond ral surface to a depth of $3 \mathrm{~mm}$ orthogonal onto the surface. This leads to a small inaccuracy in highly curved joint surfaces. Because the tibial plateau of the rabbit is relatively flat, this error is negligibly small. Second, there is the partial-volume effect, which is due to the scanner being unable to differentiate between a small amount of high-density material and a larger amount of lower density. It appears as blurring at the cartilage-bone interface. To minimize errors due to the partial-volume effect, a thin slice thickness of $1 \mathrm{~mm}$ was selected and sagittal scans perpendicular to the tibial surface were made. Third, fluctuations due to the equipment and effects due to beam hardening were avoided by calibration of the scanner against air and water before each measurement.

In the tibial plateau of the rabbit without any cartilage damage, a constant centrally located position of density maximum was found in the lateral joint surface. In the medial joint surface two density maxima areas could be determined, one of which is located anteriorly and the other posteriorly. According to the loading history this demonstrates that the main load-bearing zones are located centrally, in the zone not covered by the meniscus. In contrast to sheep, where the medial joint surface of the tibial plateau has only 1 density maximum, in rabbits there are 2 density maxima. One feasible explanation for this second density maximum on the medial surface is that there is a difference between the function of the anterior and the posterior horns of the medial meniscus. Gao et al. [20] pointed out that in the rabbit's meniscus, because of the better anchoring and the higher tensile strength of the anterior insertion ligament, the anterior horn is exposed to heavier loading than the posterior horn. Another possible explanation is the typical shape of 
the tibial plateau in the rabbit. In the sagittal plane there is an overhang of the posterior medial joint surface in relation to the proximal dorsal cortical bone. This leads to a bending stress posteriorly and might explain the second density maximum.

Mineralization subsides gradually but regularly towards the edge. The transition zone between the SBP and the metaphyseal corticalis is only sparingly mineralized. A tension analysis carried out on the tibia plate by Hayes and Carter [21], who used a finite element model, shows that when a force is centrally induced there are areas of maximum tension beneath the contact surface that regularly subside toward the edge of the tibial plateau. Furthermore, the effective forces are directed via trabeculae directly to the compact metaphyseal corticalis. This can be deduced from tension peaks in that area. Therefore, the findings made on the rabbit tibial plateau have to be understood as the result of optimal adaptation to the mechanical situation.

The analysis of BMD of the SBP showed significantly higher values in the medial tibial plateau compared to the lateral tibial plateau. This suggests that the medial tibial articular surfaces are subject to higher stress. It seems that like in humans, the point of intersection of the resulting force is in the medial compartment $[22,23]$. This finding refutes the speculation of Bendele [18], who stated that the knee joint of rabbits is more loaded laterally than medially, because lateral meniscectomy leads to more pronounced degenerative changes compared to medial meniscectomy. The reason for this is probably that in the osteoarthrosis model established by Colombo et al. [24] an additional transsection of the collateral ligament was performed.

In experimental studies, both intraindividual [25] and interindividual comparisons $[13,14]$ were used to analyze morphological parameters. It is an important fact that intraindividual and interindividual variabilities depend on the test parameter. Eckstein et al. [26] demonstrated that side differences of cartilage volume, cartilage thickness and joint surface area in the human knee joint were markedly smaller than intersubject variability. On the other hand, Boss et al. [27] found a great variation of bone density measured by quantitative CT between matched human proximal tibial metaphyses. Sone et al. [28] found a greater cortical $\mathrm{BMD}$ in the dominant leg compared to the nondominant leg, whereas no differences in trabecular BMD were noted. The higher load of the dominant limb compared to the nondominant limb was discussed as a possible explanation for side differences in bone mineral density $[29,30]$. As a result from these studies, infor- mation about intraindividual and interindividual variabilities of a test parameter is essential for planning an experimental study and for calculating the appropriate sample size. Our results clearly showed that the position of the density maxima and BMD of the SBP were identical when comparing right and left joint surfaces of the same individual. Interindividual variations of these parameters were much higher.

In summary, we have demonstrated for the first time that CT-OAM using a conventional computed tomograph allows for a reproducible and reliable determination of mineralization distribution and BMD in the SBP also in the rabbit knee. This in turn allows for an investigation of the relevance of the SBP in the context of various articular diseases also in the rabbit knee. Because of the relatively high interindividual variance of both, the position of density maxima and the BMD of the SBP, the use of the contralateral limb as control is recommended in an experimental setup.

\section{Acknowledgment}

This study was financially supported by the German Research Foundation, a nonprofit organization (DFG: AM346/1-1).

\section{Disclosure Statement}

The authors declare that they have no conflicts of interest.

References

$$
\begin{aligned}
& \text { Carter DR: Mechanical loading history and } \\
& \text { skeletal biology. J Biomech 1987;20:1095- } \\
& 1109 \text {. } \\
& 2 \text { Müller-Gerbl M, Putz R, Kenn R: Demon- } \\
& \text { stration of subchondral bone density pat- } \\
& \text { terns by three-dimensional CT osteoabsorp- } \\
& \text { tiometry as a noninvasive method for in vivo } \\
& \text { assessment of individual long-term stresses } \\
& \text { in joints. J Bone Miner Res 1992;7(suppl 2): } \\
& \text { 411-418. } \\
& 3 \text { Kummer B: Basics of Pauwels' theory of the } \\
& \text { functional adaptation of bones. Orthopäde } \\
& \text { 1995;24:387-393. } \\
& 4 \text { Jacobs CR, Eckstein F: Computer simulation } \\
& \text { of subchondral bone adaptation to mechani- } \\
& \text { cal loading in an incongruous joint. Anat } \\
& \text { Rec 1997;249:317-326. } \\
& 5 \text { Müller-Gerbl M: The subchondral bone } \\
& \text { plate. Adv Anat Embryol Cell Biol 1998, } \\
& \text { 141:III-XI, 1-134. } \\
& 6 \text { Anetzberger H, SchulzCU, Pfahler M, Refior } \\
& \text { H-J, Müller-Gerbl M: Subchondral mineral- } \\
& \text { ization patterns of the glenoid after tear of } \\
& \text { the supraspinatus. Clin Orthop 2002;404: } \\
& \text { 263-268. }
\end{aligned}
$$


7 Schulz CU, Pfahler M, Anetzberger H, Becker CR, Müller-Gerbl M, Refior H-J: The mineralization patterns at the subchondral bone plate of the glenoid cavity in healthy shoulders. J Shoulder Elbow Surg 2002;11:174-181.

$\checkmark 8$ Mochizuki Y, Natsu K, Kashiwagi K, Yasunaga $\mathrm{Y}$, Ochi M: Changes of the mineralization pattern in the subchondral bone plate of the glenoid cavity in the shoulder joints of the throwing athletes. J Shoulder Elbow Surg 2005; 14:616-619.

-9 Anetzberger H, Müller-Gerbl M, Scherer MA, Metak G, Blümel G, Putz R: Change in subchondral mineralisation after reconstruction of the anterior cruciate ligament of the sheep. Unfallchirurg 1994;97:655-660.

10 Anetzberger H, Metak G, Scherer MA, Putz R, Müller-Gerbl M: Anpassung der subchondralen Knochenplatte nach Meniskektomie als Folge einer Änderung der Spannungsverteilung. Osteologie 1995;4:224-232.

-11 Messner K, Fahlgren A, Ross I, Andersson B: Simultaneous changes in bone mineral density and articular cartilage in a rabbit meniscectomy model of knee osteoarthrosis. Osteoarthritis Cartilage 2000;8:197-206.

12 Wang SX, Laverty S, Dumitriu M, Plaas A, Grynpas MD: The effects of glucosamine hydrochloride on subchondral bone changes in an animal model of osteoarthritis. Arthritis Rheum 2007;56:1537-1548.

$\checkmark 13$ Bellido M, Lugo L, Roman-Blas JA, Casteneda S, Caeiro JR, Dapia S, Calvo E, Largo R, Herrero-Beaumont GH: Subchondral bone microstructural damage by increased remodelling aggravates experimental osteoarthritis preceded by osteoporosis. Arthritis Res Ther 2010;12:R152.
14 Sniekers YH, Weinans H, van Osch GJVM, van Leeuwen JPTM: Oestrogen is important for maintenance of cartilage and subchondral bone in a murine model of knee osteoarthritis. Arthritis Res Ther 2010;12:R182.

15 Milz S, Eckstein F, Putz R: Thickness distribution of the subchondral mineralization zone of the trochlear notch and its correlation with the cartilage thickness: an expression of functional adaptation to mechanical stress acting on the humeroulnar joint? Anat Rec 1997;248:189-197.

16 Linsenmaier U, Kersting S, Schlichtenhorst K, Putz R, Pfeifer KJ, Reiser M, Müller-Gerbl M: Functional CT imaging: load-dependent visualization of the subchondral mineralization by means of CT osteoabsorptiometry (CT-OAM). Rofo 2003;175:663-669.

17 Mühlhofer H, Ercan Y, Drews S, Matsuura M, Meissner J, Linsenmaier U, Putz R, Müller-Gerbl M: Mineralisation and mechanical strength of the subchondral bone plate of the inferior tibial facies. Surg Radiol Anat 2009; 31:237-243.

18 Bendele A: Animal models of osteoarthritis J Musculoskelet Neuronal Interact 2001;1: 363-376.

19 Kuhn JL, Goulet R-W, Pappas M, Goldstein SA: Morphometric and anisotropic symmetries of the canine distal femur. J Orthop Res 1990;8:776-780.

20 Gao J, Wei X, Messner K: Healing of the anterior attachment of the rabbit meniscus to bone. Clin Orthop 1998;348:246-258.

21 Hayes W-C, Carter D-R: Postyield behavior of subchondral trabecular bone. J Biomed Mater Res 1976;10:537-544

22 Walker PS, Erkman MJ: The role of the menisci in force transmission across the knee. Clin Orthop 1975;109:184-192.
23 Maquet PG, Pelzer GA: Evolution of the maximum stress in osteo-arthritis of the knee. J Biomech 1977;10:107-117.

24 Colombo C, Butler M, O’Byrne E, Hickman L, Swartzendruber D, Selwyn M, Steinetz B: A new model of osteoarthritis in rabbits. I. Development of knee joint pathology following lateral meniscectomy and section of the fibular collateral and sesamoid ligaments. Arthritis Rheum 1983;26:875-886.

-25 Boyd SK, Müller R, Zernicke RF: Mechanical and architectural bone adaptation in early stage experimental osteoarthritis. J Bone Miner Res 2002;17:687-694.

26 Eckstein F, Müller S, Faber S, Englmeier KH, Reiser M, Putz R: Side differences of knee joint cartilage volume, thickness and surface area, and correlation with lowerlimb dominance - an MRI based study. Osteoarthritis Cartilage 2002;10:914-921.

$>27$ Boos C, Fink K, Stomberg P, Köller W, Igl BW, Russlies M: The intraindividual agreement of the bone density of the human proximal tibia. Ann Anat 2007;189:515-519.

28 Sone T, Imai Y, Joo YI, Onodera S, Tomomitsu T, Fukunaga M: Side-to-side differences in cortical bone mineral density of tibiae in young male athletes. Bone 2006;38: 708-713.

29 Min JY, Min KB, Paek D, Cho SI: Side differences in the bone density of the distal radius and calcaneus in Koreans aged $4-86$ years. J Clin Densitom 2007;10:184-188.

30 Ireland A, Korhonen M, Heinonen A, Suominen H, Baur C, Stevens S, Degens H, Rittweger J: Side-to-side differences in bone strength in master jumpers and sprinters. J Musculoskelet Neuronal Interact 2011;11: 298-305. 